\title{
Rate Allocation in Wireless Sensor Networks with Network Lifetime Requirement
}

\author{
Y. Thomas Hou* Yi Shi \\ The Bradley Department of \\ Electrical and Computer Engineering \\ Virginia Tech, Blacksburg, VA \\ \{thou,yshi\}@vt.edu
}

\author{
Hanif D. Sherali \\ The Grado Department of \\ Industrial and Systems Engineering \\ Virginia Tech, Blacksburg, VA \\ hanifs@vt.edu
}

\begin{abstract}
An important performance consideration for wireless sensor networks is the amount of information collected by all the nodes in the network over the course of network lifetime. Since the objective of maximizing the sum of rates of all the nodes in the network can lead to a severe bias in rate allocation among the nodes, we advocate the use of lexicographical max-min (LMM) rate allocation for the nodes. To calculate the LMM rate allocation vector, we develop a polynomial-time algorithm by exploiting the parametric analysis (PA) technique from linear programming (LP), which we call serial LP with Parametric Analysis (SLP-PA). We show that the SLP-PA can be also employed to address the so-called LMM node lifetime problem much more efficiently than an existing technique proposed in the literature. More important, we show that there exists an elegant duality relationship between the LMM rate allocation problem and the LMM node lifetime problem. Therefore, it is sufficient to solve any one of the two problems and important insights can be obtained by inferring duality results for the other problem.
\end{abstract}

\section{Categories and Subject Descriptors}

C.2.1 [Network Architecture and Design]: Wireless communication

\section{General Terms}

Algorithms, Performance, Theory

\section{Keywords}

Wireless sensor networks, energy constraint, network capacity, rate allocation, lexicographic max-min, node lifetime, parametric analysis, linear programming, flow routing

${ }^{*}$ This research has been supported in part by NSF ANI-0312655, ONR N00014-03-1-0521, and the Woodrow W. Everett, Jr. SCEEE Development Fund.

Permission to make digital or hard copies of all or part of this work for personal or classroom use is granted without fee provided that copies are not made or distributed for profit or commercial advantage and that copies bear this notice and the full citation on the first page. To copy otherwise, to republish, to post on servers or to redistribute to lists, requires prior specific permission and/or a fee.

MobiHoc'04, May 24-26, 2004, Roppongi, Japan.

Copyright 2004 ACM 1-58113-849-0/04/0005 ...\$5.00.

\section{INTRODUCTION}

Wireless sensor networks consist of battery-powered nodes that are endowed with a multitude of sensing modalities including multimedia (e.g., video, audio) and scalar data (e.g., temperature, pressure, light, magnetometer, infrared). Although there have been significant improvements in processor design and computing, advances in battery technology still lag behind, making energy resource considerations the fundamental challenge in wireless sensor networks. As a consequence, there have been active research efforts on exploring performance limits of wireless sensor networks. These performance limits include, among others, network capacity (see e.g., [12]) and network lifetime (see e.g., [7, 8]). Network capacity typically refers to the maximum amount of bit volume that can be successfully delivered to the base-station ("sink node") by all the nodes in the network, while network lifetime refers to the maximum time limit that nodes in the network remain alive until one or more nodes drain up their energy.

In this paper, we consider an important overarching problem that encompasses both performance metrics. In particular, we study the network capacity problem under a given network lifetime requirement. Specifically, for a wireless sensor network where each node is provisioned with an initial energy, if all nodes are required to live up to a certain lifetime criterion, what is the maximum amount of bit volume that can be generated by the entire network? At first glance, it appears desirable to maximize the sum of rates from all the nodes in the network, subject to the condition that each node can meet the network lifetime requirement. Mathematically, this problem can be formulated as a linear programming (LP) problem (see Section 2.2) within which the objective function is defined as the sum of rates over all the nodes in the network and the constraints are: (1) flow balance is preserved at each node, and (2) the energy constraint at each node can be met for the given network lifetime requirement. However, the solution to this problem shows (see Section 5) that although the network capacity (i.e., the sum of bit rates over all nodes) is maximized, there exists a severe bias in the rate allocation among the nodes. In particular, those nodes that consume the least amount of power on their data path toward the base-station will be allocated with much more bit rates than other nodes in the network. Consequently, the data collection behavior for the entire network only favors certain nodes that have this property, while other nodes will be unfavorably penalized with much smaller bit rates.

The fairness issue associated with the network capacity maximization objective calls for a careful consideration in the rate allocation among the nodes. In this paper, we investigate the rate 
allocation problem in an energy-constrained sensor network for a given network lifetime requirement. Our objective is to achieve a certain measure of optimality in the rate allocation that takes into account both fairness and bit rate maximization. We advocate to use of the so-called Lexicographic Max-Min (LMM) criterion [14], which maximizes the bit rates for all the nodes until one or more nodes reach their energy limit for the given network lifetime requirement. At first level, the smallest rate among all the nodes is maximized. We continue to maximize the second level of smallest rate and so forth. The LMM rate allocation criterion is appealing since it addresses both fairness and efficiency (i.e., bit rate maximization) in an energy-constrained network.

A naive approach to the LMM rate allocation problem would be to apply a max-min-like iterative procedure. Under this naive approach, successive LPs are employed to calculate the maximum rate at each level based on the available energy for the remaining nodes, until all nodes use up their energy. We call this naive approach "serial LP" (SLP). We show that, although SLP appears intuitive, unfortunately it gives an incorrect solution. To understand how this could happen, we must understand a fundamental difference between the LMM rate allocation problem described here and the classical max-min rate allocation in [3]. Under the LMM rate allocation problem, the rate allocation problem is implicitly coupled with a flow routing problem, while under the classical maxmin rate allocation, there is no routing problem involved since the routes for all flows are fixed. As it turns out, for the LMM rate allocation problem, any iterative rate allocation approach that requires energy reservation at each iteration is incorrect. This is because, unlike max-min, which addresses only the rate allocation problem with fixed routes and yields a unique solution at each iteration, for the LMM rate allocation problem, starting from the first iteration, there usually exist non-unique flow routing solutions corresponding to the same rate allocation at each level. Consequently, each of these flow routing solutions will yield different available energy levels on the remaining nodes for future iterations and so forth, leading to a different rate allocation vector, which usually does not coincide with the optimal LMM rate allocation vector.

In this paper, we develop an efficient polynomial-time algorithm to solve the LMM rate allocation problem. We exploit the so-called parametric analysis (PA) technique [2] at each rate level to determine the minimum set of nodes that must deplete their energy. We call this approach serial LP with PA (SLP-PA). In most cases when the problem is non-degenerate, the SLP-PA algorithm is extremely efficient and only requires quadratic time complexity in determining the minimum node set for each rate level. Even for the rare case when the problem is degenerate, the SLP-PA algorithm is still much more efficient than the slack variable (SV)-based approach proposed in [6], due to fewer number of LPs involved at each rate level.

We also extend the PA technique for the LMM rate allocation problem to address the so-called maximum node lifetime curve problem in [6], which we call LMM node lifetime problem. We show that the SLP-PA approach is much more efficient than the slack variable (SV)-based approach described in [6]. More importantly, we show that there exists a simple and elegant duality relationship between the LMM rate allocation problem and the LMM node lifetime problem. As a result, it is sufficient to solve only one of these two problems and important insights can be obtained by inferring duality results for the other problem.

The remainder of this paper is organized as follows. In Section 2, we describe the network and energy model, and formulate

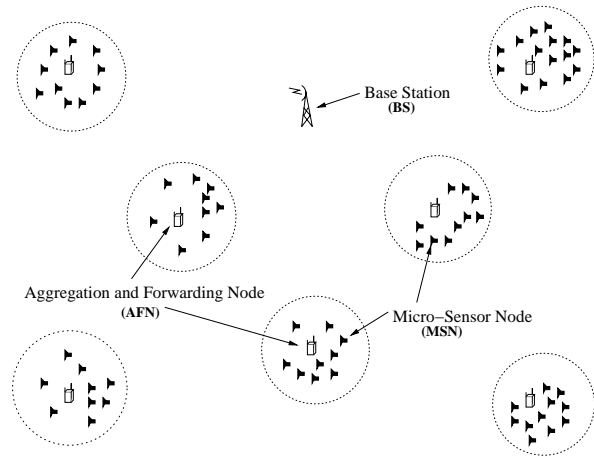

(a) Physical topology.

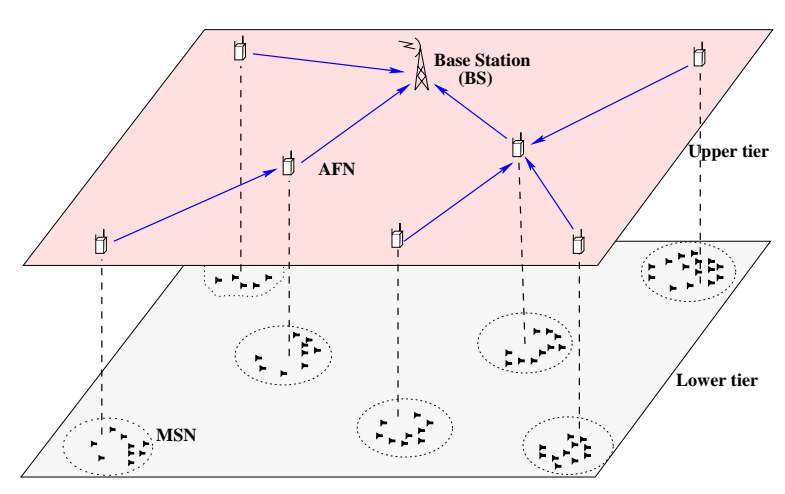

(b) A hierarchical view.

Figure 1: Reference architecture for two-tiered wireless sensor networks.

the LMM rate allocation problem. Section 3 presents our SLP-PA algorithm to the LMM rate allocation problem. In Section 4, we introduce the LMM node lifetime problem and apply the SLP-PA algorithm to solve it. We also show how the LMM rate allocation problem and the LMM node lifetime problem are linked by a duality relationship. Numerical results are presented in Section 5. Section 6 reviews related work and Section 7 concludes this paper.

\section{SYSTEM MODELING AND PROBLEM FORMULATION}

We consider a two-tiered architecture for wireless sensor networks. The two-tiered network architecture is motivated by recent advances in distributed source coding (DSC) [9, 15, 17], which is capable of removing redundancy in information collected among neighboring sensors without inter-sensor communications. Figures 1(a) and (b) show the physical and hierarchical network topology for such a network, respectively. There are three types of nodes in the network, namely, micro-sensor nodes (MSNs), agregation and forwarding nodes (AFNs), and a base-station (BS). The MSNs can be application-specific sensor nodes (e.g., temperature sensor nodes (TSNs), pressure sensor nodes (PSNs), and video sensor nodes (VSNs)) and they constitute the lower tier of the network. They are deployed in groups (or clusters) at strategic locations for 
surveillance or monitoring applications. The MSNs are small and low-cost. The objective of an MSN is very simple: Once triggered by an event (e.g., detection of motion or biological/chemical agents), it starts to capture live information (e.g., video), which it sends directly to the local AFN. ${ }^{1}$

For each cluster of MSNs, there is one AFN, which is different from an MSN in terms of physical properties and functions. The primary functions of an AFN are: (1) data aggregation (or "fusion") for data flows from the local cluster of MSNs, and (2) forwarding (or relaying) the aggregated information to the next hop AFN (toward the base-station). For data fusion, an AFN analyzes the content of each data stream (e.g., video) it receives, from which it composes a complete scene by exploiting the correlation among each individual data stream from the MSNs. An AFN also serves as a relay node for other AFNs to carry traffic toward the base-station. Although an AFN is expected to be provisioned with much more energy than an MSN, it also consumes energy at a substantially higher rate (due to wireless communication over large distances). Consequently, an AFN has a limited lifetime. Upon depletion of energy at an AFN, we expect that the coverage for the particular area under surveillance is lost, despite the fact that some of the MSNs within the cluster may still have remaining energy. ${ }^{2}$

The third component in the two-tiered architecture is the basestation. The base-station is, essentially, the sink node for data streams from all the AFNs in the network. In this investigation, we assume that there is sufficient energy resource available at the basestation and thus there is no energy constraint at the base-station. In summary, the main functions of the lower tier MSNs are data acquisition and compression while the upper-tier AFNs are used for data fusion and relaying information to the base-station.

\subsection{Power Consumption Model}

For AFN $i$, we assume that the aggregated bit rate collected by its local MSNs after data fusion is $g_{i}, i=1,2, \cdots, N$. These collected local bit streams must be routed toward the base-station. Our objective is to maximize the $g_{i}$ values according to the LMM criterion (see Definition 1 below) under a given network lifetime requirement.

For an AFN, energy consumption due to wireless communication (i.e., receiving and transmitting) has been considered the dominant factor in power consumption [1]. The power dissipation at a radio transmitter can be modeled as:

$$
p_{t}(i, k)=c_{i k} \cdot f_{i k},
$$

where $p_{t}(i, k)$ is the power dissipated at AFN $i$ when it is transmitting to node $k, f_{i k}$ is the rate transmitted from $\mathrm{AFN} i$ to node $k, c_{i k}$ is the power consumption cost of radio link $(i, k)$ and is given by

$$
c_{i k}=\alpha+\beta \cdot d_{i k}^{m},
$$

where $\alpha$ is a distance-independent constant term, $\beta$ is a coefficient term associated with the distance-dependent term, $d_{i k}$ is the distance between these two nodes, and $m$ is the path loss index, with $2 \leq m \leq 4$ [18]. Typical values for these parameters are $\alpha=50$ $\mathrm{nJ} / \mathrm{b}$ and $\beta=0.0013 \mathrm{pJ} / \mathrm{b} / \mathrm{m}^{4}$ (for $m=4$ ) [10]. ${ }^{3}$ Since the power level of an AFN's transmitter can be used to control the distance coverage of an AFN (see, e.g., [16, 19, 21]), different network flow

\footnotetext{
${ }^{1}$ Due to the small distance between an MSN and its local AFN, multi-hop routing among the MSNs may not be necessary.

${ }^{2}$ We assume that each MSN can only forward information to its local AFN for processing (e.g., video fusion).

${ }^{3}$ In this paper, we use $m=4$ in all of our numerical results.
}

routing topologies can be formed by adjusting the power level of each AFN's transmitter.

The power dissipation at a receiver can be modeled as [18]:

$$
p_{r}(i)=\rho \cdot \sum_{k \neq i} f_{k i},
$$

where $\sum_{k \neq i} f_{k i}$ (in $\mathrm{b} / \mathrm{s}$ ) is the rate of the received data stream at AFN $i$. A typical value for the parameter $\rho$ is $50 \mathrm{~nJ} / \mathrm{b}$ [10].

\subsection{The LMM Rate Allocation Problem}

Before we formulate the LMM rate allocation problem, let us revisit the maximum capacity problem (with "bias" in rate allocation) that was described in Section 1. For a network with $N$ AFNs, suppose that the rate of AFN $i$ is $g_{i}$, and that the initial energy at this node is given by $e_{i}(i=1,2, \cdots, N)$. For a given network lifetime requirement $T$ (i.e., each AFN must remain alive for at least time duration $T$ ), the maximum information capacity that the network can collect can be formulated as the following linear programming (LP).

$$
\begin{aligned}
\frac{\text { MaxCap: }}{\text { s.t. }} & \operatorname{Max} \sum_{i=1}^{N} g_{i} \\
f_{i B}+\sum_{k \neq i} f_{i k}-\sum_{m \neq i} f_{m i}=g_{i} & (1 \leq i \leq N) \\
\sum_{m \neq i} \rho f_{m i} T+\sum_{k \neq i} c_{i k} f_{i k} T+c_{i B} f_{i B} T \leq e_{i} & (1 \leq i \leq N) \\
f_{i k}, f_{i B} \geq 0 & (1 \leq i, k \leq N, k \neq i)
\end{aligned}
$$

where $f_{i k}$ and $f_{i B}$ are data rates transmitted from AFN $i$ to AFN $k$ and from AFN $i$ to the base-station $B$, respectively. The set of constraints in (4) are the flow balance equations: they state that, the total bit rate transmitted by AFN $i$ is equal to the total bit rate received by AFN $i$ from other AFNs, plus the bit rate generated locally at AFN $i\left(g_{i}\right)$. The set of constraints in (5) are the energy constraints: they state that, for a given network lifetime requirement $T$, the energy required in communications (i.e., in transmitting and receiving all these data) cannot exceed the initial energy provisioning level.

Note that $f_{m i}, f_{i k}, f_{i B}$, and $g_{i}$ are variables and that $T$ is a constant (representing a given network lifetime requirement). MaxCap is a standard LP formulation that can be solved by a polynomial algorithm [2]. Unfortunately, as we shall see in the numerical results (Section 5), the solution to this MaxCap problem lends itself into an extreme bias toward AFNs whose data paths consume the least amount of power toward the base-station. Consequently, although the network capacity is maximized over the network lifetime $T$, the corresponding bit rate allocation among the AFNs (i.e., the $g_{i}$ values) only favors those AFNs that have this property, while other AFNs are unfavorably allocated with much smaller (even close to 0 ) bit rates. As a result, the effectiveness of the network in performing information collection or surveillance could be severely compromised.

To address this fairness issue, we advocate the so-called lexicographic max-min (LMM) rate allocation strategy [14] in this paper, which has some similarity to the max-min rate allocation in data networks [3]. ${ }^{4}$ Under LMM rate allocation, we start with the objective of maximizing the bit rate for all the nodes until one or more nodes reach their energy constraint capacities for the given network lifetime requirement. Given that the first level of the smallest rate allocated among the nodes is maximized, we continue to

${ }^{4}$ However, there is significant difference between max-min and LMM, which we will discuss shortly. 
maximize the second level of rate for the remaining nodes that still have available energy, and so forth. More formally, denote $\mathbf{r}=$ $\left[r_{1}, r_{2}, \cdots, r_{N}\right]$ as the sorted version (i.e., $r_{1} \leq r_{2} \leq \cdots \leq r_{N}$ ) of the rate vector $\mathbf{g}=\left[g_{1}, g_{2}, \cdots, g_{N}\right]$, with $g_{i}$ corresponding to the rate of node $i$. We then have the following definition for an LMM rate allocation.

\section{DEFINITION 1. (LMM-optimal Rate Allocation)}

For a given network lifetime requirement $T$, a sorted rate vector $\mathbf{r}=\left[r_{1}, r_{2}, \cdots, r_{N}\right]$ yields an LMM-optimal rate allocation if and only iffor any other sorted rate allocation vector $\hat{r}=\left[\hat{r}_{1}, \hat{r}_{2}, \cdots, \hat{r}_{N}\right]$ with $\hat{r}_{1} \leq \hat{r}_{2} \leq \cdots \leq \hat{r}_{N}$, there exists a $k, 1 \leq k \leq N$, such that $r_{i}=\hat{r}_{i}$ for $1 \leq i \leq k-1$ and $r_{k}>\hat{r}_{k}$.

Based on the LMM-optimal definition, we can calculate the first level optimal rate $\lambda_{1}=r_{1}$ easily through the following LP.

$\operatorname{Max} \lambda_{1}$

s.t.

$$
\begin{array}{rlrl}
f_{i B}+\sum_{k \neq i} f_{i k}-\sum_{m \neq i} f_{m i}-\lambda_{1} & =0 & & (1 \leq i \leq N) \\
\sum_{m \neq i} \rho T f_{m i}+\sum_{k \neq i} c_{i k} T f_{i k}+c_{i B} T f_{i B} & \leq e_{i} & & (1 \leq i \leq N) \\
f_{i k}, f_{i B} & \geq 0 & (1 \leq i, k \leq N, k \neq i)
\end{array}
$$

Although the first level bottleneck rate $\lambda_{1}$ is easy to obtain, calculating the subsequent bottleneck rates are quite challenging. As discussed in Section 1, a naive approach that applies an iterative LP procedure to calculate the desired rate allocations is incorrect. This is because there is a fundamental difference in the nature of the LMM rate allocation problem described here and the classical max-min rate allocation problem in [3]. The LMM rate allocation problem implicitly couples a flow routing problem (i.e., a determination of the $f_{i k}$ and $f_{i B}$ for the entire network), while the classical max-min rate allocation explicitly assumes that the routes for all the flows are given a priori and fixed. Moreover, for the LMM rate allocation problem, starting from the first iteration, there usually exist non-unique flow routing solutions corresponding to the same maximum rate level. Consequently, each of these flow routing solutions, once chosen, will yield different remaining energy levels on the nodes for future iterations and so forth, leading to a different rate vector, which usually does not coincide with the LMM-optimal rate vector. Therefore, any iterative rate allocation algorithm that requires energy reservation among the nodes during each iteration is unlikely to give a correct LMM rate allocation (see Section 5 for numerical example). In the next section, we present an efficient (polynomial time) algorithm to solve the LMM rate allocation problem correctly without requiring any energy reservation during each iteration.

\section{A SERIAL LP ALGORITHM BASED ON PARAMETRIC ANALYSIS}

\subsection{Problem Formulation}

To solve the LMM rate allocation problem, we first perform the following problem formulation. Table 1 lists the notation used in this paper. Suppose that the sorted rate vector $\mathbf{r}=\left[r_{1}, r_{2}, \cdots, r_{N}\right]$ with $r_{1} \leq r_{2} \leq \cdots \leq r_{N}$ is LMM-optimal. To keep track of

\begin{tabular}{|c|c|}
\hline \multicolumn{2}{|c|}{ General Notation to the LMM-Rate and LMM-Lifetime problems } \\
\hline$N$ & The total number of AFNs in the network \\
\hline$e_{i}$ & The initial energy at AFN $i$ \\
\hline$\rho$ & The power consumption coefficient for receiving data \\
\hline$c_{i k}$ & The power consumption coefficient for transmitting \\
\hline$\left(\right.$ or $\left.c_{i B}\right)$ & data from AFN $i$ to AFN $k$ (or the base-station $B$ ) \\
\hline$n$ & $\begin{array}{l}\text { The number of distinct elements in the sorted } \\
\text { LMM-optimal rate/lifetime vector }\end{array}$ \\
\hline$S_{i}$ & $\begin{array}{l}\text { The minimum set of nodes that reach their energy } \\
\text { constraint limits at } i \text {-th level }\end{array}$ \\
\hline$\hat{S}_{i}$ & $\begin{array}{l}\text { The set of all possible AFNs that may reach their } \\
\text { energy constraint limits at } i \text {-th level, } S_{i} \subset \hat{S}_{i}\end{array}$ \\
\hline $\begin{array}{c}V_{i k} \\
\text { (or } V_{i B} \text { ) }\end{array}$ & $\begin{array}{l}\text { The total volume from AFN } i \text { to AFN } k- \\
\text { (or the base-station } B \text { ) }\end{array}$ \\
\hline $\begin{array}{c}f_{i k} \\
\left.\text { (or } f_{i B}\right)\end{array}$ & $\begin{array}{l}\text { The rate from AFN } i \text { to AFN } k \\
\text { (or the base-station } B \text { ) }\end{array}$ \\
\hline$x$ & The optimal solution to LMM-Rate/LMM-Lifetime \\
\hline$w$ & $\begin{array}{l}\text { The optimal solution to dual problem of LMM-Rate } \\
\text { or LMM-Lifetime }\end{array}$ \\
\hline$b$ & $\begin{array}{l}\text { The right-hand-side (RHS) of LMM-Rate } \\
\text { or LMM-Lifetime }\end{array}$ \\
\hline$I_{i}$ & $\begin{array}{l}\text { A column vector having a single } 1 \text { element } \\
\text { corresponding to node } i \text { in Eq. (10) or Eq. (15) and } \\
0 \text { for all other elements }\end{array}$ \\
\hline $\mathcal{B}$ & $\begin{array}{l}\text { The columns corresponding to the basic variables in } \\
\text { LMM-Rate/LMM-Lifetime }\end{array}$ \\
\hline $\mathcal{Z}$ & $\begin{array}{l}\text { The columns corresponding to the non-basic variables } \\
\text { in LMM-Rate/LMM-Lifetime }\end{array}$ \\
\hline$c_{\mathcal{B}}$ & $\begin{array}{l}\text { The parameters in objective function corresponding } \\
\text { to the basic variables of LMM-Rate/LMM-Lifetime }\end{array}$ \\
\hline$c_{\mathcal{Z}}$ & $\begin{array}{l}\text { The parameters in objective function corresponding } \\
\text { to the non-basic variables of LMM-Rate } \\
\text { or LMM-Lifetime }\end{array}$ \\
\hline$x_{\mathcal{B}}$ & $\begin{array}{l}\text { Part of optimal solution corresponding to the basic } \\
\text { variables of LMM-Rate/LMM-Lifetime }\end{array}$ \\
\hline$x_{\mathcal{Z}}$ & $\begin{array}{l}\text { Part of optimal solution corresponding to the } \\
\text { non-basic variables of LMM-Rate/LMM-Lifetime }\end{array}$ \\
\hline \multicolumn{2}{|r|}{ Symbols used for the LMM-Rate problem } \\
\hline$T$ & The network lifetime requirement \\
\hline$g_{i}$ & The local bit rate collected at AFN $i$ \\
\hline$r_{i}$ & $\begin{array}{l}\text { The } i \text {-th element in the sorted LMM-optimal rate } \\
\text { vector, where } r_{1} \leq r_{2} \leq \cdots \leq r_{N}\end{array}$ \\
\hline$\lambda_{i}$ & $\begin{array}{l}\text { The } i \text {-th rate level in the sorted LMM-optimal rate } \\
\text { vector, i.e., } \lambda_{1}\left(=r_{1}\right)<\lambda_{2}<\cdots<\lambda_{n}\left(=r_{N}\right)\end{array}$ \\
\hline$\delta_{i}$ & $=\lambda_{i}-\lambda_{i-1}$, the difference between $\lambda_{i}$ and $\lambda_{i-1}$ \\
\hline \multicolumn{2}{|r|}{ Symbols used for the LMM-Lifetime problem } \\
\hline$\overline{g_{i}}$ & The rate requirement at $\mathrm{AFN} i$ \\
\hline$t_{i}$ & The node lifetime at AFN $i$ \\
\hline$\tau_{i}$ & $\begin{array}{l}\text { The } i \text {-th element in the sorted LMM-optimal } \\
\text { lifetime vector, where } \tau_{1} \leq \tau_{2} \leq \cdots \leq \tau_{N}\end{array}$ \\
\hline$\mu_{i}$ & $\begin{array}{l}\text { The } i \text {-th drop point in the sorted LMM-optimal } \\
\text { lifetime vector, i.e., } \mu_{1}\left(=r_{1}\right)<\mu_{2}<\cdots<\mu_{n}\left(=r_{N}\right)\end{array}$ \\
\hline$\zeta_{i}$ & $=\mu_{i}-\mu_{i-1}$, the difference between $\mu_{i}$ and $\mu_{i-1}$ \\
\hline
\end{tabular}
distinct rates, we remove all repetitive elements in this vector and
Table 1: Notation 
rewrite it as $\left[\lambda_{1}, \lambda_{2}, \cdots, \lambda_{n}\right]$ such that $\lambda_{1}<\lambda_{2}<\cdots<\lambda_{n}$, where $\lambda_{1}=r_{1}, \lambda_{n}=r_{N}$, and $n \leq N$. For each $\lambda_{i}$, denote $S_{i}$, $i=1,2, \cdots, n$, as the corresponding set of nodes that use up their energy at this rate. Clearly, $\sum_{i=1}^{n}\left|S_{i}\right|=|S|=N$, where $S$ denotes the set of all $N$ nodes. The key to the LMM rate allocation problem is to find the correct values $\lambda_{1}, \lambda_{2}, \cdots, \lambda_{n}$ and the corresponding set $S_{1}, S_{2}, \cdots, S_{n}$, respectively.

To formulate this problem into an iterative form, we define $\lambda_{0}=$ 0 and $S_{0}=\emptyset$. Furthermore, denote $\delta_{l}=\lambda_{l}-\lambda_{l-1}$. Starting with $l=1(1 \leq l \leq n)$, we have an iterative optimization problem as follows.

$\operatorname{Max} \delta_{l}$

s.t.

$$
\begin{aligned}
& f_{i B}+\sum_{k \neq i} f_{i k}-\sum_{m \neq i} f_{m i}-\delta_{l}=\lambda_{l-1} \quad\left(i \notin \bigcup_{h=0}^{l-1} S_{h}\right) \\
& f_{i B}+\sum_{k \neq i} f_{i k}-\sum_{m \neq i} f_{m i}=\lambda_{h} \quad\left(i \in \bigcup_{h=1}^{l-1} S_{h}\right) \\
& \sum_{m \neq i} \rho T f_{m i}+\sum_{k \neq i} c_{i k} T f_{i k}+c_{i B} T f_{i B} \leq e_{i} \quad\left(i \notin \bigcup_{h=0}^{l-1} S_{h}\right) \\
& \sum_{m \neq i} \rho T f_{m i}+\sum_{k \neq i} c_{i k} T f_{i k}+c_{i B} T f_{i B}=e_{i} \quad\left(i \in \bigcup_{h=1}^{l-1} S_{h}\right) \\
& f_{i k}, f_{i B} \geq 0 \quad(1 \leq i, k \leq N, k \neq i)
\end{aligned}
$$

Note that for $l=1$, the constraints (7) and (9) do not exist. For $2 \leq l \leq n$, constraints (7) and (9) are for those nodes that have already reached their LMM rate allocation during the previous $l-1$ iterations. In particular, the set of constraints in (7) say that the sum of in-coming and local data rates are equal to the out-going data rates for each node with its LMM-optimal rate $\lambda_{h}, 1 \leq h<l$. The set of constraints in (9) say that for these nodes that have already reached their LMM-optimal rate, the total energy consumed for communications has reached their initial energy provisioning at these nodes. On the other hand, the constraints in (6) and (8) are for the remaining nodes that have not yet reached their LMMoptimal rate. Specifically, the set of constraints in (6) state that, for these nodes that have not yet reached their energy constraint levels, the sum of in-coming and local data rates are equal to the out-going data rates. Note that the objective function is to maximize the additional rate $\delta_{l}$ for these nodes. Furthermore, for these nodes, the set of constrains in (8) state that the total energy consumed for communications should be upper bounded by the initial energy provisioning.

To facilitate our later discussion on duality results in Section 4, we further re-formulate above LP. In particular, we multiply both sides of (6) and (7) by $T$ (which is a constant representing a given network lifetime requirement) and denote $V_{i B}=f_{i B} T, V_{i k}=$ $f_{i k} T, V_{m i}=f_{m i} T$. Intuitively, $V_{i k}$ and $V_{i B}$ represent the bit volume that is transferred from node $i$ to $k$ and from node $i$ to $B$, respectively, during lifetime $T$. We obtain the following problem formulation.

\section{LMM-Rate: Max $\delta_{l}$}

s.t.

$$
\begin{array}{r}
V_{i B}+\sum_{k \neq i} V_{i k}-\sum_{m \neq i} V_{m i}-\delta_{l} T=\lambda_{l-1} T \quad\left(i \notin \bigcup_{h=1}^{l-1} S_{h}\right) \\
V_{i B}+\sum_{k \neq i} V_{i k}-\sum_{m \neq i} V_{m i}=\lambda_{h} T \quad\left(i \in \bigcup_{h=1}^{l-1} S_{h}\right)
\end{array}
$$

$$
\begin{array}{rlrl}
\sum_{m \neq i} \rho V_{m i}+\sum_{k \neq i} c_{i k} V_{i k}+c_{i B} V_{i B} & \leq e_{i} & \left(i \notin \bigcup_{h=1}^{l-1} S_{h}\right) \\
\sum_{m \neq i} \rho V_{m i}+\sum_{k \neq i} c_{i k} V_{i k}+c_{i B} V_{i B} & =e_{i} & \left(i \in \bigcup_{h=1}^{l-1} S_{h}\right) \\
V_{i k}, V_{i B} \geq 0 & (1 \leq i, k \leq N, k \neq i)
\end{array}
$$

The above LP formulation can be rewritten in the form Max $c x$, s.t. $A x=b$ and $x \geq 0$, the dual problem for which is Min $w b$, s.t. $w A \geq c$ with $w$ being unrestricted in sign [2]. Both can be solved by standard LP techniques (e.g., [2]). Although a solution to the LMM-Rate problem gives the optimal solution for $\delta_{l}$ at iteration $l$, it remains to determine the minimum set of nodes corresponding to this $\delta_{l}$, which is the key difficulty in the LMM rate allocation problem. In the rest of this section, we exploit the parametric analysis technique [2] to determine the minimum node set at each rate.

\subsection{Minimum Node Set Determination}

Denote $\hat{S}_{l}\left(\hat{S}_{l} \neq \emptyset\right)$ the set of nodes for which the constraints (8) are binding at the $l$-th iteration for the LMM-Rate problem, i.e., $\hat{S}_{l}$ include all the nodes that achieve equality in (8) at iteration $l$. Although it is certain that at least one of the nodes in $\hat{S}_{l}$ belong to $S_{l}$ (the minimum node set for rate $\lambda_{l}$ ), for other nodes in $\hat{S}_{l}$, it may still be possible to further increase their rates under alternative flow routing solutions. In other words, if $\left|\hat{S}_{l}\right|=1$, then we must have $S_{l}=\hat{S}_{l}$; otherwise, we must determine the minimum node set $S_{l}$ $\left(S_{l} \subseteq \hat{S}_{l}\right)$ that achieves the LMM-optimal rate allocation.

We find that the so-called parametric analysis (PA) technique [2] is a powerful technique to address this problem. The main idea of PA is to investigate how an infinitesimal perturbation on some components of the LMM-Rate problem can affect the objective function. In particular, considering a small increase on the right-handside (RHS) of (10), i.e., changing $b_{i}$ to $b_{i}+\epsilon_{i}$, where $\epsilon_{i}>0$, node $i$ belongs to the minimum node set $S_{l}$ if and only if $\frac{\partial^{+} \delta_{l}}{\partial \epsilon_{i}}(0)<0$. That is, node $i$ belongs to the minimum node set $S_{l}$ if and only if a small increase in node $i$ 's rate (in terms of total volume generated at node $i$ ) leads to a decrease in the objective function.

To compare $\frac{\partial^{+} \delta_{l}}{\partial \epsilon_{i}}(0)$ with 0 , we apply an important duality results from LP theory. If $x$ and $w$ are the respective optimal solution to the primal and dual problems, then based on the parametric duality property [2], we have

$$
\frac{\partial^{+} \delta_{l}}{\partial \epsilon_{i}}(0)=\frac{\partial^{+}(c x)}{\partial b_{i}}\left(b_{i}\right) \leq w_{i}
$$

Recall that these $w_{i}$ can be easily obtained at the same time when we solve the primal LP problem. Note that by the nature of the problem, we have $w_{i} \leq 0$ for an optimal dual solution. Therefore, if we find that $w_{i}<0$, then we can determine immediately that node $i$ must belong to the minimum node set $S_{l}$. On the other hand, if we find that $w_{i}=0$, it is not clear whether $\frac{\partial^{+} \delta_{l}}{\partial \epsilon_{i}}(0)$ is strictly negative or 0 and further analysis is thus needed.

For each node $i$ with $w_{i}=0$, we must perform a complete PA to see whether a perturbation (i.e., tiny increase) on the RHS of (10) will result in any change in the objective function. If there is no change, then we can determine that node $i$ does not belong to the minimum node set $S_{l}$; otherwise, node $i$ belongs to $S_{l}$. Assume that the optimal solution is $\left(x_{\mathcal{B}}, x_{\mathcal{Z}}\right)$, where $x_{\mathcal{B}}$ and $x_{\mathcal{Z}}$ denote the set of basic and non-basic variables; $\mathcal{B}$ and $\mathcal{Z}$ denote the columns corresponding to the basic and non-basic variables. $c_{\mathcal{B}}$ and $c_{\mathcal{Z}}$ denote the objective function coefficient vectors for the basic and non- 
basic variables; and $q$ denotes the objective value. Then we have the corresponding canonical equations as follows

$$
\begin{aligned}
q+\left(c_{\mathcal{B}}^{t} \mathcal{B}^{-1} \mathcal{Z}-c_{\mathcal{Z}}^{t}\right) x_{\mathcal{Z}} & =c_{\mathcal{B}}^{t} \mathcal{B}^{-1} b \\
x_{\mathcal{B}}+\left(\mathcal{B}^{-1} \mathcal{Z}\right) x_{\mathcal{Z}} & =\mathcal{B}^{-1} b .
\end{aligned}
$$

If $b$ is replaced by $b+\epsilon_{i} I_{i}$, where the column vector $I_{i}$ has a single 1 element corresponding to node $i$ in the set of constraints (10) while all the other elements are 0 , then the only change due to this perturbation is that $\mathcal{B}^{-1} b$ will be replaced by $\mathcal{B}^{-1}\left(b+\epsilon_{i} I_{i}\right)$. Consequently, the objective value for the current basis becomes $c_{\mathcal{B}}^{t} \mathcal{B}^{-1}\left(b+\epsilon_{i} I_{i}\right)$. As long as $\mathcal{B}^{-1}\left(b+\epsilon_{i} I_{i}\right)$ is nonnegative, the current basis remains optimal. Denote $\bar{b}=\mathcal{B}^{-1} b, \mathcal{B}_{i}^{-1}=\mathcal{B}^{-1} I_{i}$, and let $\hat{\epsilon}_{i}$ be an upper bound for $\epsilon_{i}$ such that the current basis remains optimal. We have

$$
\hat{\epsilon}_{i}=\min _{j}\left\{\frac{\bar{b}_{j}}{-\mathcal{B}_{i j}^{-1}}: \mathcal{B}_{i j}^{-1}<0\right\} .
$$

If $\hat{\epsilon}_{i}>0$, the optimal objective value varies according to $c_{\mathcal{B}}^{t} \mathcal{B}^{-1}(b+$ $\left.\epsilon_{i} I_{i}\right)$ for $0<\epsilon_{i} \leq \hat{\epsilon}_{i}$. Since $w=c_{\mathcal{B}}^{t} \mathcal{B}^{-1}$ and $w_{i}=0$, we have $c_{\mathcal{B}}^{t} \mathcal{B}^{-1} I_{i}=w_{i}=0$. Thus, the objective value will not change for $\epsilon_{i} \in\left(0, \hat{\epsilon}_{i}\right]$, and consequently, the rate for node $i$ can be increased beyond the current $\lambda_{l}$ value. That is, node $i$ does not belong to the minimum node set $S_{l}$.

For most problems in practice, the above procedure is sufficient to determine whether or not node $i$ belongs to the minimum node set $S_{l}$ for all $i \in \hat{S}_{l}$. But in the rare event where $\hat{\epsilon}_{i}=0$, the problem is degenerate. To develop a polynomial-time algorithm, denote $W_{l}$ as the set of all nodes with $w_{i}<0$ and $U_{l}$ as the set of all nodes with $w_{i}=0$ and $\hat{\epsilon}_{i}=0$. Then we solve the following LP to maximize the slack variables (SV) for nodes in $U_{l}$.

$$
\begin{array}{rc}
\frac{\text { MSV: }}{\text { s.t. }} \operatorname{Max} \sum_{i \in U_{l}} \epsilon_{i} \\
V_{i B}+\sum_{k \neq i} V_{i k}-\sum_{m \neq i} V_{m i}-\epsilon_{i} T=\lambda_{l} T & \left(i \in U_{l}\right) \\
V_{i B}+\sum_{k \neq i} V_{i k}-\sum_{m \neq i} V_{m i}=\lambda_{h} T & \left(i \in \bigcup_{h=1}^{l-1} S_{h}\right) \\
V_{i B}+\sum_{k \neq i} V_{i k}-\sum_{m \neq i} V_{m i}=\lambda_{l} T & \left(i \notin U_{l} \bigcup_{h=1}^{l-1} S_{h}\right) \\
\sum_{m \neq i} \rho V_{m i}+\sum_{k \neq i} c_{i k} V_{i k}+c_{i B} V_{i B}=e_{i} & \left(i \in U_{l} \bigcup W_{l} \bigcup_{h=1}^{l-1} S_{h}\right) \\
\sum_{m \neq i} \rho V_{m i}+\sum_{k \neq i} c_{i k} V_{i k}+c_{i B} V_{i B} \leq e_{i} & \left(i \notin U_{l} \bigcup W_{l} \bigcup_{h=1}^{l-1} S_{h}\right) \\
V_{i k}, V_{i B}, \epsilon_{i} \geq 0 & (1 \leq i, k \leq N, k \neq i)
\end{array}
$$

If the optimal objective function is 0 , then we conclude that no node in $U_{l}$ can have a positive $\epsilon_{i}$. That is, these nodes should all belong to $S_{l}$ and we have $S_{l}=W_{l}+U_{l}$. On the other hand, if the optimal objective function is positive, then some nodes $i \in U_{l}$ must have positive $\epsilon_{i}$ values and these nodes therefore do not belong to the minimum node set $S_{l}$. Consequently, we can remove these nodes from $U_{l}$. If $U_{l} \neq \emptyset$, we move on to solve another MSV. This procedure will terminate when the optimal objective function value is 0 or $U_{l}=\emptyset$.

To ensure that MSV determinate the minimum node set correctly, we need the following lemma. The proof is give in [11].
Lemma 1. (The Minimum Node Set is Unique.)

The minimum node set for each rate level under the LMM-optimal rate allocation is unique.

In a nutshell, the complete PA procedure to determine whether a node $i \in \hat{S}_{l}$ belongs to the minimum node set $S_{l}$ can be summarized as follows.

\section{AlgorithM 1. (Minimum Node Set Determination with PA)}

1. Initialize sets $W_{l}=\emptyset$ and $U_{l}=\emptyset$.

2. For each node $i \in \hat{S}_{l}$,

(a) If $w_{i}<0$, then $W_{l}=W_{l} \cup\{i\}$.

(b) Otherwise (i.e., $w_{i}=0$ ), compute $\bar{b}=\mathcal{B}^{-1} b, \mathcal{B}_{i}^{-1}=$ $\mathcal{B}^{-1} I_{i}$, and $\hat{\epsilon}_{i}$ according to $(12)$. If $\hat{\epsilon}_{i}=0$, then $U_{l}=U_{l}+\{i\}$.

3. If $U_{l}=\emptyset$, then $S_{l}=W_{l}$ and stop; else set up the MSV problem and solve it.

4. If the optimal objective value in MSV is 0 , then $S_{l}=W_{l}+U_{l}$ and stop; else remove all nodes $i$ with $\epsilon_{i}>0$ from the set $U_{l}$ and go to Step 3.

\subsection{Optimal Flow Routing for LMM Rate Allocation}

Once we solve the LMM rate allocation problem, the corresponding optimal flow routing can be easily obtained by dividing the total bit volume on each link $\left(V_{i k}\right.$ or $\left.V_{i B}\right)$ by $T$, i.e.,

$$
\begin{gathered}
f_{i k}=\frac{V_{i k}}{T}, \\
f_{i B}=\frac{V_{i B}}{T},
\end{gathered}
$$

where $T$ is the given network lifetime requirement. Although the LMM-optimal rate allocation is unique, it is important to note that the corresponding optimal flow routing solution is not unique. This is because upon the completion of the LMM rate allocation problem (i.e., upon finding $\left[\lambda_{1}, \lambda_{2}, \cdots, \lambda_{n}\right]$ ), there usually exist nonunique bit volume solutions ( $V_{i k}$ and $V_{i B}$ values) corresponding to the same LMM-optimal rate allocation. This result is summarized in the following lemma.

LEMMA 2. The optimal flow routing solution corresponding to the LMM rate allocation may not be unique.

\subsection{Complexity Analysis}

We now analyze the complexity of the SLP-PA algorithm in solving the LMM rate allocation problem. First we consider the complexity of finding each node's rate and the total bit volume transmitted along each link. At each stage, we solve an LP problem, both its primal and dual have a complexity of $O\left(n_{A}{ }^{3} L\right)$ [2], where $n_{A}$ is the number of constraints or variables in the problem, whichever is larger, and $L$ is the number of binary bits required to store the data. Since the number of variables is $O\left(N^{2}\right)$ and is larger than the number of constraints (which is $O(N)$ ), the complexity of solving the LP is $O\left(N^{6} L\right)$. After solving an LP at each stage, we need to determine whether or not a node that just reached its energy binding constraint belongs to the minimum node set for this stage. Note that $w$ and $\hat{b}=\mathcal{B}^{-1} b$ can be readily obtained when we solve the 
primal LP problem. To determine whether a node, say $i$, belongs to the minimum node set, we examine $w_{i}$. If $w_{i}<0$, then node $i$ belongs to the minimum node set and the complexity is $O(1)$. On the other hand, if $w_{i}=0$, we need to further examine whether $\hat{\epsilon}_{i}>0$ or not. Based on (12), the computation for $\hat{\epsilon}_{i}$ is $O(N)$. So at each stage, the complexity in PA for each node is $O(N)$. The total complexity of PA at each stage for the node set is thus $\left|\hat{S}_{l}\right| \cdot O(N)$ or $O(N \cdot N)=O\left(N^{2}\right)$. Thus, the complexity at each stage is $O\left(N^{6} L\right)+O\left(N^{2}\right)=O\left(N^{6} L\right)$. As there are at most $N$ stages, the overall complexity is $O\left(N^{7} L\right)$.

We now analyze the complexity for the degenerate case. Upon the completion of Step 2 in Algorithm 1, we denote $U_{l}^{(0)}=U_{l}$. Since we need to solve at most $\left|U_{l}^{(0)}-S_{l}\right|$ LPs, the complexity is $\left|U_{l}^{(0)}-S_{l}\right| \cdot O\left(N^{6} L\right)$ or $O\left(N \cdot N^{6} L\right)=O\left(N^{7} L\right)$. Hence, the complexity at each stage is $O\left(N^{6} L\right)+O\left(N^{2}\right)+O\left(N^{7} L\right)=$ $O\left(N^{7} L\right)$. Since there are at most $N$ stages, the overall complexity is $O\left(N^{8} L\right)$.

The complexity in finding the optimal flow routing is bounded by the number of radio links in the network, which is $O\left(N^{2}\right)$. Hence the overall complexity is $O\left(N^{7} L\right)+O\left(N^{2}\right)=O\left(N^{7} L\right)$ for the non-degenerate case and $O\left(N^{8} L\right)+O\left(N^{2}\right)=O\left(N^{8} L\right)$ for the degenerate case. Under either case, the computational complexity is polynomial. ${ }^{5}$

\section{EXTENSION TO LMM NODE LIFETIME PROBLEM AND DUALITY THEOREM}

In this section, we present two important extensions for our results in Section 3. First, we show that our SLP-PA algorithm can be used to solve the maximum node lifetime curve problem in [6], which we define as the LMM node lifetime problem. We show that the SLP-PA algorithm is a much more efficient approach than the one proposed in [6]. Second, we show that there exists an elegant duality relationship between the LMM rate allocation problem and the LMM node lifetime problem. Consequently, important results and insights can be drawn by simply solving one of the two problems.

\subsection{The LMM-optimal Node Lifetime Problem and Solution}

The LMM node lifetime problem considers the following scenario. For a network with $N$ AFNs, with a given local bit rate $g_{i}$ (fixed) and initial energy $e_{i}$ for AFN $i, i=1,2, \cdots, N$, how can we maximize the network lifetime for all AFNs in the network? In other words, the LMM node lifetime problem not only considers how to maximize the network lifetime until the first AFN runs out of energy, but also the time for all the AFNs in the network.

More formally, for each AFN $i$, denote the corresponding lifetime as $t_{i}, i=1,2, \cdots, N$. Note that $g_{i}$ are fixed here, while $t_{i}$ are the optimization variables, which are different from the LMM rate allocation problem that we studied in the last section. Denote $\left[\tau_{1}, \tau_{2}, \cdots, \tau_{N}\right]$ as the sorted sequence of the $t_{i}$ values in nondecreasing order. Then LMM-optimal node lifetime can be defined as follows.

DEFINITION 2. (LMM-optimal Node Lifetime) A sorted node lifetime vector $\left[\tau_{1}, \tau_{2}, \cdots, \tau_{N}\right]$ with $\tau_{1} \leq \tau_{2} \leq$

${ }^{5}$ Note that our analysis here give a loose upper bound for time complexity. In practice, the actual running time for LP implementation is much faster than its upper bound. $\cdots \leq \tau_{N}$ is LMM-optimal if and only if for any other sorted node lifetime vector $\left[\hat{\tau}_{1}, \hat{\tau}_{2}, \cdots, \hat{\tau}_{N}\right]$ with $\hat{\tau}_{1} \leq \hat{\tau}_{2} \leq \cdots \leq \hat{\tau}_{N}$, there exists a $k, 1 \leq k \leq N$, such that $\tau_{i}=\hat{\tau}_{i}$ for $1 \leq i \leq k-1$ and $\tau_{k}>\hat{\tau}_{k}$

Solution. It should be clear that, under the LMM-optimal node lifetime objective, we must maximize the time until a set of nodes use up their energy (which is also called a drop point in [6]) while minimizing the number of nodes that drain up their energy at each drop point. We now show that the SLP-PA algorithm developed for the LMM rate allocation problem can be directly applied to solve the LMM node lifetime problem.

Suppose that $\left[\tau_{1}, \tau_{2}, \cdots, \tau_{N}\right]$ with $\tau_{1} \leq \tau_{2} \leq \cdots \leq \tau_{N}$ is LMM-optimal. To keep track of distinct node lifetimes (or drop points) in this vector, we remove all repetitive elements in the vector and rewrite it as $\left[\mu_{1}, \mu_{2}, \cdots, \mu_{n}\right]$ such that $\mu_{1}<\mu_{2}<\cdots<$ $\mu_{n}$, where $\mu_{1}=\tau_{1}, \mu_{n}=\tau_{N}$, and $n \leq N$. Corresponding to these drop points, denote $S_{1}, S_{2}, \cdots, S_{n}$ as the sets of nodes that drain up their energy at drop points $\mu_{1}, \mu_{2}, \cdots, \mu_{n}$, respectively. Then $\left|S_{1}\right|+\left|S_{2}\right|+\cdots+\left|S_{n}\right|=|S|=N$, where $S$ denotes the set of all $N$ AFNs in the network. The problem is to find the LMM-optimal values of $\mu_{1}, \mu_{2}, \cdots, \mu_{n}$ and the corresponding sets $S_{1}, S_{2}, \cdots, S_{n}$.

Similar to the LMM rate allocation problem, the LMM node lifetime problem can be formulated as an iterative optimization problem as follows. Denote $\mu_{0}=0, S_{0}=\emptyset$, and $\zeta_{l}=\mu_{l}-\mu_{l-1}$. Starting from $l=1$, we solve the following LP iteratively.

LMM-Lifetime: $\operatorname{Max} \zeta_{l}$

s.t.

$$
\begin{array}{rc}
V_{i B}+\sum_{k \neq i} V_{i k}-\sum_{m \neq i} V_{m i}-\zeta_{l} g_{i}=\mu_{l-1} g_{i} & \left(i \notin \bigcup_{h=0}^{l-1} S_{h}\right) \\
V_{i B}+\sum_{k \neq i} V_{i k}-\sum_{m \neq i} V_{m i}=\mu_{h} g_{i} & \left(i \in \bigcup_{h=0}^{l-1} S_{h}\right) \\
\sum_{m \neq i} \rho V_{m i}+\sum_{k \neq i} c_{i k} V_{i k}+c_{i B} V_{i B} \leq e_{i} & \left(i \notin \bigcup_{h=0}^{l-1} S_{h}\right) \\
\sum_{m \neq i} \rho V_{m i}+\sum_{k \neq i} c_{i k} V_{i k}+c_{i B} V_{i B}=e_{i} & \left(i \in \bigcup_{h=0}^{l-1} S_{h}\right) \\
V_{i k}, V_{i B}, \zeta_{l} \geq 0 & (1 \leq i, k \leq N, k \neq i)
\end{array}
$$

Comparing the LMM-Lifetime problem here to the LMM-Rate problem that we studied in Section 3.1, we find that they are exactly of the same form. The only differences are that under the LMMLifetime problem, the local bit rates $g_{i}$ are constants and the node lifetimes $\tau_{i}$ are variables (subject to optimization), while under the LMM-Rate problem, the $g_{i}$ are variables (subject to optimization) and the node lifetimes are all identical $(T), i=1,2, \cdots, N$. Since the mathematical formulation for the two problems are identical, we can apply the SLP-PA algorithm to solve the LMM node lifetime problem as well.

The only issue that we need to be concerned about is the optimal flow routing solution corresponding to the LMM-optimal lifetime vector. The optimal flow routing solution here is not as simple as that for the LMM rate allocation problem, which merely involves a simple division (see Eqs. (13) and (14)). We refer readers to [11] for an $O\left(N^{4}\right)$ algorithm to obtain an optimal flow routing solution for the LMM-optimal lifetime vector. Similar to Lemma 2, the optimal flow routing solution corresponding to the LMM node lifetime problem may not be unique. 
Complexity Comparison. In [6], Brown et al. studied the LMM node lifetime problem under the so-called "maximum node lifetime curve" problem. They also made an important contribution by developing the first procedure to solve this problem correctly. A key step in their procedure is the use of multiple independent LP calculations to determine the minimum node set at each drop point, which we call serial LP with slack variable analysis (SLP$\mathrm{SV}$ ). Although this approach solves the LMM node lifetime problem correctly, its computational complexity (potentially exponential) remains an issue to be resolved.

On the other hand, the SLP-PA algorithm developed in this paper is strictly polynomial and is computationally more efficient than the SLP-SV approach. To understand the difference between the two, we take a closer look on the computational complexity of the SLPSV approach in [6]. First, SLP-SV needs to keep track of each subflow along its route from the source node toward the base-station. Such a flow-based (or more precisely, sub-flow based) approach could make the size of the LP coefficient matrix exponential, which leads to an exponential-time algorithm [2]. ${ }^{6}$

Second, even if a link-based LP formulation such as ours is adopted in [6], the computational efficiency of the SV-based approach is still worse than the SLP-PA algorithm. This is because at each stage, the SV-based approach must solve several additional LPs (up to $\left.\left|\hat{S}_{l}-S_{l}\right|\right)$ to determine $S_{l}$, which is in contrast to the simpler PA under the SLP-PA algorithm $\left(O\left(N^{2}\right)\right)$. Even for the degenerate case, the number of additional LPs under the SLP-PA algorithm is at most $\left|U_{l}^{(0)}-S_{l}\right|{ }^{7}$ which is still no more than $\left|\hat{S}_{l}-S_{l}\right|$.

Finally, we discuss a hybrid link-flow approach mentioned in [6]. In this approach, link-based formulations are used for sub-flows. This leads to a much fewer number of variables than those for the flow-based approach. But this approach still requires sub-flow accounting and results in an order of magnitude more constraints than the link-based approach in SLP-PA. Although this approach solves the LMM node lifetime problem in polynomial-time (e.g., by using interior point methods [2]), the overall complexity is still orders of magnitude higher than that under the SLP-PA algorithm. Furthermore, the burden of solving additional LPs to determine whether a node belongs to the minimum node set still remains.

\subsection{Duality Theorem}

In this section, we present an elegant and powerful result showing that there is an underlying duality relationship between the LMM rate allocation problem and the LMM node lifetime problem. Consequently, the solutions and insights obtained for one problem can be "mirrored" to the other problem.

To start with, we denote $\mathcal{P}_{R}$ as the LMM rate allocation problem where we have $N$ AFNs in the network and all nodes have a common given lifetime requirement $T$ (constant). Denote $g_{i}$ as the LMM-optimal rate allocation for node $i$ under $\mathcal{P}_{R}, i=$ $1,2, \cdots, N$. Similarly, we denote $\mathcal{P}_{L}$ as the LMM node lifetime problem where all nodes have the same local bit rate $R$ (constant). Denote $t_{i}$ as the LMM node lifetime for node $i$ under $\mathcal{P}_{L}$, $i=1,2, \cdots, N$. Then the following theorem shows how the solution to one problem can be used to obtain the solution to the other.

\footnotetext{
${ }^{6}$ Incidentally, the revised simplex method proposed in [6] is not as efficient as the polynomial-time algorithm described in [2] and is itself exponential.

${ }^{7}$ Recall that $U_{l}^{(0)}$ denotes $U_{l}$ upon the completion of Step 2 in Algorithm 1.
}

Table 2: Duality relationship between LMM rate allocation problem $\mathcal{P}_{R}$ and LMM node lifetime problem $\mathcal{P}_{L}$.

\begin{tabular}{|c|c|}
\hline LMM rate allocation $\left(\mathcal{P}_{R}\right)$ & LMM node lifetime $\left(\mathcal{P}_{L}\right)$ \\
\hline$g_{i}$ (optimization variable) & $g_{i}=R$ \\
\hline$t_{i}=T$ (constant) & $t_{i}$ (optimization variable) \\
\hline \multicolumn{2}{|c|}{ Total bit volume at AFN $i: g_{i} \cdot T=t_{i} \cdot R$} \\
\hline
\end{tabular}

\section{THEOREM 1. (Duality Theorem)}

For a given node lifetime requirement $T$ for all nodes under problem $\mathcal{P}_{R}$ and a given local bit rate $R$ for all nodes under problem $\mathcal{P}_{L}$, we have the following relationship between the solutions to the LMM rate allocation problem $\mathcal{P}_{R}$ and the LMM node lifetime problem $\mathcal{P}_{L}$.

(i) Suppose that we have solved problem $\mathcal{P}_{R}$ and obtained the LMM-optimal rate allocation $g_{i}$ for each node $i(i=1,2, \cdots, N)$. Then under $\mathcal{P}_{L}$, the $L M M$ node lifetime $t_{i}$ for node $i$ is

$$
t_{i}=\frac{g_{i} T}{R} .
$$

(ii) Suppose that we have solved problem $\mathcal{P}_{L}$ and obtained the LMM-optimal node lifetime $t_{i}$ for each node $i(i=1,2, \cdots, N)$. Then under $\mathcal{P}_{R}$, the LMM rate allocation $g_{i}$ for node $i$ is

$$
g_{i}=\frac{t_{i} R}{T} .
$$

Table 2 shows the duality relationship between solutions to problems $\mathcal{P}_{R}$ and $\mathcal{P}_{L}$.

Proof. We prove (i) and (ii) in Theorem 1 separately.

(i) We organize our proof into two parts. First, we show that $t_{i}$ are feasible node lifetimes in terms of flow balance and energy constraints on each node $i(i=1,2, \cdots, N)$. Then we show that it is indeed the LMM-optimal node lifetime.

Feasibility. Since we have obtained the solution to problem $\mathcal{P}_{R}$, we have one feasible flow routing solution for sending bit streams $g_{i}, i=1,2, \cdots, N$, to the base-station. Under problem $\mathcal{P}_{R}$, the bit volumes ( $V_{i j}$ and $V_{i B}$ values) must meet the following equalities under the LMM-optimal rate allocation:

$$
\begin{gathered}
V_{i B}+\sum_{1 \leq k \leq N, k \neq i} V_{i k}-\sum_{1 \leq m \leq N, m \neq i} V_{m i}=g_{i} T, \\
\sum_{1 \leq m \leq N, m \neq i} \rho V_{m i}+\sum_{1 \leq k \leq N, k \neq i} c_{i k} V_{i k}+c_{i B} V_{i B}=e_{i} .
\end{gathered}
$$

Now replacing $g_{i} T$ by $t_{i} R$, we see that the same bit volume solution under $\mathcal{P}_{R}$ yields a feasible bit volume solution to the node lifetime problem under $\mathcal{P}_{L}$. Consequently, we can obtain the flow routing solution to problem $\mathcal{P}_{L}$ under the bit volume solution to problem $\mathcal{P}_{L}[11]$ and this verifies that $t_{i}, i=1,2, \cdots, N$, is a feasible solution to problem $\mathcal{P}_{L}$.

Optimality. To prove that the $t_{i}, i=1,2, \cdots, N$, obtained via (16) are indeed LMM-optimal for problem $\mathcal{P}_{L}$, we sort $g_{i}$, $i=1,2, \cdots, N$, under problem $\mathcal{P}_{R}$ in non-decreasing order and denote it as $\left[r_{1}, r_{2}, \cdots, r_{N}\right]$. We also introduce a node index $I=$ $\left[i_{1}, i_{2}, \cdots, i_{N}\right]$ for $\left[r_{1}, r_{2}, \cdots, r_{N}\right]$. For example, $i_{3}=7$ means that $r_{3}$ actually corresponds to the rate of AFN 7, i.e., $r_{3}=g_{7}$.

Since $t_{i}$ is proportional to $g_{i}$ through the relationship $\left(t_{i}=\frac{T}{R}\right.$. $\left.g_{i}\right)$, listing $t_{i}, i=1,2, \cdots, N$, according to $I=\left[i_{1}, i_{2}, \cdots, i_{N}\right]$ 
Table 3: Node coordinates for the 10-AFN network.

\begin{tabular}{|c|c||c|c|}
\hline$i$ & $\left(x_{i}, y_{i}\right)$ (in meters) & $i$ & $\left(x_{i}, y_{i}\right)$ (in meters) \\
\hline 1 & $(400,-320)$ & 6 & $(-500,100)$ \\
\hline 2 & $(300,440)$ & 7 & $(-400,0)$ \\
\hline 3 & $(-300,-420)$ & 8 & $(420,120)$ \\
\hline 4 & $(320,-100)$ & 9 & $(200,140)$ \\
\hline 5 & $(-120,340)$ & 10 & $(220,-340)$ \\
\hline
\end{tabular}

will also yield a sorted (in non-decreasing order) lifetime list, denoted as $\left[\tau_{1}, \tau_{2}, \cdots, \tau_{N}\right]$. We now prove that $\left[\tau_{1}, \tau_{2}, \cdots, \tau_{N}\right]$ is indeed LMM-optimal for problem $\mathcal{P}_{L}$.

Our proof is based on contradiction. Suppose that $\left[\tau_{1}, \tau_{2}, \cdots, \tau_{N}\right]$ is not LMM-optimal for problem $\mathcal{P}_{L}$. Assume that the LMMoptimal lifetime vector to problem $\mathcal{P}_{L}$ is $\left[\hat{\tau}_{1}, \hat{\tau}_{2}, \cdots, \hat{\tau}_{N}\right]$ (sorted in non-decreasing order) with the corresponding node index being $\hat{I}=\left[\hat{i}_{1}, \hat{i}_{2}, \cdots, \hat{i}_{N}\right]$. Then, by Definition 2 , there exists a $k$ such that $\hat{\tau}_{j}=\tau_{j}$ for $1 \leq j \leq k-1$ and $\hat{\tau}_{k}>\tau_{k}$.

We now claim that if $\hat{t}_{i}, i=1,2, \cdots, N$, is a feasible solution to problem $\mathcal{P}_{L}$, then $\hat{g}_{i}$ obtained via $\hat{g}_{i}=\frac{\hat{t}_{i} R}{T}, i=1,2, \cdots, N$, is also a feasible solution to problem $\mathcal{P}_{R}$. The proof for this claim follows identically as above. Using this result, we can obtain a corresponding feasible solution $\left[\hat{r}_{1}, \hat{r}_{2}, \cdots, \hat{r}_{N}\right]$ with $\hat{r}_{i}=\frac{\hat{t}_{i} R}{T}$ and the node index $\hat{I}$ for problem $\mathcal{P}_{R}$. Hence we have $\hat{r}_{j}=\frac{\hat{\tau}_{j} R}{T}=$ $\frac{\tau_{j} R}{T}=r_{j}$ for $1 \leq j \leq k-1$ but $\hat{r}_{k}=\frac{\hat{\tau}_{k} R}{T}>\frac{\tau_{k} R}{T}=r_{k}$. That is, $\left[r_{1}, r_{2}, \cdots, r_{N}\right]$ is not LMM-optimal and this leads to a contradiction.

(ii) The proof for this part is similar to the above proof for (i) and is thus omitted here.

This duality relationship can offer important insights on system performance issues, in addition to providing solutions to the LMM rate allocation and the LMM node lifetime problems. For example, in Section 1, we pointed out the potential bias (fairness) issue associated with the network capacity maximization objective (i.e., sum of rates from all nodes). It is interesting to see that there is a dual fairness issue under the node lifetime problem. In particular, the objective of maximizing the sum of node lifetimes among all nodes also leads to a bias (or fairness) problem because this objective would only favor those nodes that consume energy at a small rate. As a result, certain nodes will have much larger lifetimes while some other nodes will be penalized with much smaller lifetimes.

\section{NUMERICAL INVESTIGATION}

In this section, we use numerical results to illustrate our SLP-PA algorithm to the LMM rate allocation problem and compare it with other approaches. We also use numerical results to illustrate the duality relationship between the LMM rate allocation problem and the LMM node lifetime problem.

Due to space limitation, we will show results for a network of 10 AFNs. More results for network of larger size are available in [11]. In this 10 -AFN network, the base-station $B$ is located at the origin while the locations for the 10 AFNs are randomly generated over a $1000 \mathrm{~m} \times 1000 \mathrm{~m}$ square area (see Fig. 2 and Table 3).

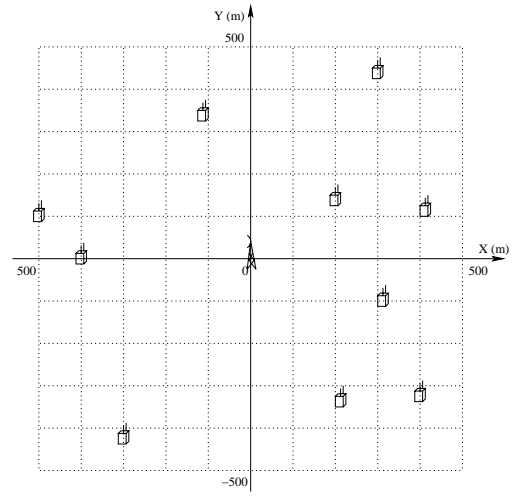

Figure 2: Network topology used in the numerical investigation.

Table 4: Rate allocation under the SLP-PA, SLP, and MaxCap approaches for the 10-AFN Network.

\begin{tabular}{|c||c|c||c|c||c|c|}
\hline \multicolumn{1}{|c||}{$\begin{array}{c}i \text { (Sorted } \\
\begin{array}{c}\text { Node } \\
\text { Index) }\end{array}\end{array}$} & $\begin{array}{c}c|| c|| c \mid \\
\text { SLP-PA } \\
(\mathrm{kb} / \mathrm{s})\end{array}$ & AFN & $\begin{array}{c}r_{i} \\
(\mathrm{~kb} / \mathrm{s})\end{array}$ & AFN & $\begin{array}{c}r_{i} \\
(\mathrm{~kb} / \mathrm{s})\end{array}$ & AFN \\
\hline 1 & 0.1023 & 3 & 0.1023 & 1 & 0.0553 & 2 \\
\hline 2 & 0.1023 & 6 & 0.1023 & 2 & 0.0627 & 3 \\
\hline 3 & 0.1023 & 7 & 0.1023 & 3 & 0.0646 & 1 \\
\hline 4 & 0.1536 & 5 & 0.1023 & 6 & 0.0658 & 6 \\
\hline 5 & 0.2941 & 1 & 0.1023 & 7 & 0.1222 & 8 \\
\hline 6 & 0.2941 & 2 & 0.1536 & 5 & 0.1653 & 10 \\
\hline 7 & 0.2941 & 4 & 0.1536 & 8 & 0.1736 & 7 \\
\hline 8 & 0.2941 & 8 & 0.1536 & 10 & 0.2628 & 5 \\
\hline 9 & 0.2941 & 9 & 0.6563 & 4 & 0.3513 & 4 \\
\hline 10 & 0.2941 & 10 & 0.6563 & 9 & 1.2398 & 9 \\
\hline
\end{tabular}

\subsection{SLP-PA Algorithm to the LMM Rate Allocation Problem}

We will compare SLP-PA with the naive approach (see Section 2.2) that uses a serial LP "blindly" to solve the LMM rate allocation problem. We call this naive approach Serial LP (SLP). As discussed in Section 2.2, the naive SLP approach requires energy reservation at each stage and will not give the correct final solution to the LMM rate allocation problem.

We will also compare our SLP-PA algorithm with the MaximumCapacity (MaxCap) approach (see Section 2.2). As discussed in the beginning of Section 2.2, the rate allocation under the MaxCap approach can be extremely biased and favors only those AFNs that consume the least power along their data paths toward the basestation.

We assume that the initial energy at each AFN is $50 \mathrm{~kJ}$ and that under the LMM rate allocation problem, the network lifetime requirement is 100 days. The power consumption behaviors for transmission and reception are defined in (1) and (3), respectively.

Table 4 shows the rate allocation for the AFNs under each approach, which is also plotted in Fig. 3. The "sorted node index" corresponds the sorted rates among the AFNs in non-decreasing order.

Clearly, among the three rate allocation approaches, only the rate allocation under SLP-PA meets the LMM-optimal rate allocation definition (see Definition 1) when compared with the rate allocation under SLP and MaxCap. Specifically, comparing SLP- 


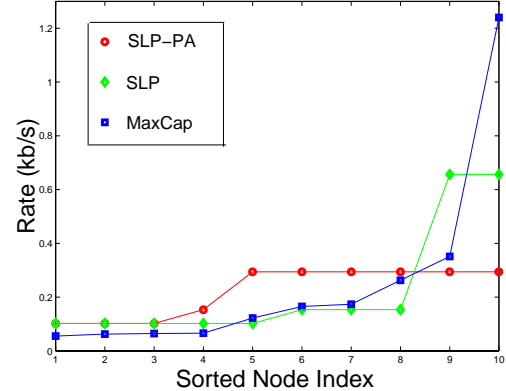

Figure 3: Rate allocation under the SLP-PA, SLP, and MaxCap approaches for a 10-AFN network .

PA with SLP, we have $r_{1}^{\text {SLP-PA }}=r_{1}^{\mathrm{SLP}}, r_{2}^{\mathrm{SLP}-\mathrm{PA}}=r_{2}^{\mathrm{SLP}}$, $r_{3}^{\mathrm{SLP}-\mathrm{PA}}=r_{3}^{\mathrm{SLP}}$, and $r_{4}^{\mathrm{SLP}-\mathrm{PA}}>r_{4}^{\mathrm{SLP}}$; comparing SLP-PA with MaxCap, we have $r_{1}^{\text {SLP-PA }}>r_{1}^{\text {MaxCap }}$.

We also observe, as expected, a severe bias in the rate allocation under the MaxCap approach. In particular, $r_{10}$ alone accounts for over $48 \%$ of the sum of total rates among all the AFNs. Comparing the three approaches, we have $r_{1}^{\mathrm{SLP}-\mathrm{PA}}=r_{1}^{\mathrm{SLP}}>r_{1}^{\text {MaxCap }}$ and $r_{10}^{\mathrm{SLP}-\mathrm{PA}}<r_{10}^{\mathrm{SLP}}<r_{10}^{\mathrm{MaxCap}}$. In other words, the rate allocation vector under the SLP-PA algorithm has the smallest rate difference between the smallest rate $\left(r_{1}\right)$ and the largest rate $\left(r_{10}\right)$, i.e., $r_{10}-r_{1}$, among the three approaches. In addition, although $r_{1}^{\mathrm{SLP}-\mathrm{PA}}=r_{1}^{\mathrm{SLP}}$ for the first level rate allocation, the minimum node set for $r_{1}^{\text {SLP-PA }}$ is smaller than the minimum node set for $r_{1}^{\mathrm{SLP}}$, i.e., $\left|S_{1}^{\mathrm{SLP}-\mathrm{PA}}\right|=3<\left|S_{1}^{\mathrm{SLP}}\right|=5$. This confirms that the naive SLP approach cannot offer the correct solution to the LMM rate allocation problem.

\subsection{Duality Results}

We now use numerical results to verify the duality relationship between the LMM rate allocation problem $\left(\mathcal{P}_{R}\right)$ and the LMM node lifetime problem $\left(\mathcal{P}_{L}\right)$ (see Section 4.2). Again, we use the 10-AFN network configurations in Fig. 2. The coordinates for each AFN under the 10-AFN network are listed in Table 3. We assume that the initial energy at each AFN is $50 \mathrm{~kJ}$ and that the network lifetime requirement under the LMM rate allocation problem is $T=100$ days. Under $\mathcal{P}_{L}$, we assume the local bit rate for all AFNs are $R=0.2 \mathrm{~kb} / \mathrm{s}$.

To verify the duality relationship (Theorem 1 ), we perform the following calculations. First, we solve the LMM rate allocation problem $\left(\mathcal{P}_{R}\right)$ and the LMM node lifetime problem $\left(\mathcal{P}_{L}\right)$ independently with the above initial conditions using the SLP-PA algorithm. Consequently, we obtain the LMM-optimal rate allocation ( $g_{i}$ for each AFN $i$ ) under $\mathcal{P}_{R}$ and the LMM-optimal node lifetime $\left(t_{i}\right.$ for each AFN $i$ ) under $\mathcal{P}_{L}$. Then we compute $T \cdot g_{i}$ and $R \cdot t_{i}$ separately for each AFN $i$ and examine if they are equal to each other.

The results for the LMM-optimal rate allocation $\left(g_{i}, i=1,2, \cdots\right.$, $10)$ and the LMM-optimal node lifetime $\left(t_{i}, i=1,2, \cdots, 10\right)$ for the 10-AFN network are shown in Table 5. We find that $T \cdot g_{i}$ and $R \cdot t_{i}$ are exactly equal for all AFNs, precisely as we would expect under Theorem 1.
Table 5: Numerical results verifying the duality relationship $T$. $g_{i}=R \cdot t_{i}$ between the LMM rate allocation problem $\left(\mathcal{P}_{R}\right)$ and the LMM node lifetime problem $\left(\mathcal{P}_{L}\right)$ for the 10-AFN network.

\begin{tabular}{|c||c|r||r|r|}
\hline \multicolumn{1}{|c||}{ AFN } & \multicolumn{1}{c||}{$\mathcal{P}_{R}(T=100$ days $)$} & \multicolumn{2}{c|}{$\mathcal{P}_{L}(R=0.2 \mathrm{~kb} / \mathrm{s})$} \\
\cline { 2 - 5 } & $g_{i}$ & $T \cdot g_{i}$ & $t_{i}$ & $R \cdot t_{i}$ \\
\hline 1 & 0.2941 & 29.41 & 147.07 & 29.41 \\
\hline 2 & 0.2941 & 29.41 & 147.07 & 29.41 \\
\hline 3 & 0.1023 & 10.23 & 51.17 & 10.23 \\
\hline 4 & 0.2941 & 29.41 & 147.07 & 29.41 \\
\hline 5 & 0.1536 & 15.36 & 76.79 & 15.36 \\
\hline 6 & 0.1023 & 10.23 & 51.17 & 10.23 \\
\hline 7 & 0.1023 & 10.23 & 51.17 & 10.23 \\
\hline 8 & 0.2941 & 29.41 & 147.07 & 29.41 \\
\hline 9 & 0.2941 & 29.41 & 147.07 & 29.41 \\
\hline 10 & 0.2941 & 29.41 & 147.07 & 29.41 \\
\hline
\end{tabular}

\section{RELATED WORK}

Due to energy constraints in wireless sensor networks, there has been active research on exploring the performance limits of such networks. These performance limits include, among others, network capacity and network lifetime. Network capacity typically refers to the maximum amount of bit volume that can be successfully delivered to the base-station ("sink node") by all the nodes in the network, where network lifetime refers to the maximum time that the nodes in the network remain alive before one or more nodes deplete their energy.

The network capacity problem and network lifetime problem have so far been studied disjointly in the literature. For example, in [12], the problem of how to maximize network capacity via routing was studied. While, in many other efforts (see, e.g., [4, 5, 8, $13,22]$ ), the focus was on how to maximize the time until the first node drains up its energy.

In this paper, we study the important overarching problem that considers both network capacity and network lifetime. Under the LMM rate allocation problem, we studied how to maximize rate allocations for all the nodes in the network under a given network lifetime requirement. Under the LMM node lifetime problem, we studied how to maximize the lifetime for all nodes when the local bit rate for each node is given a priori. The LMM rate allocation criterion effectively mitigates the unfairness issue when the objective is to maximize the total bit volume generated by the network. Although the LMM rate allocation is somewhat similar to the classical max-min strategy [3], there is a fundamental difference between the two. In particular, the LMM rate allocation problem implicitly embeds (or couples) a flow routing problem within rate allocation, while under the classical max-min rate allocation, there is no routing problem involved since the routes for all flows are fixed. Due to this coupling of flow routing and rate allocation, a solution approach (i.e., SLP-PA) to the LMM rate allocation problem is much more challenging than that for the classical max-min.

In [20], Srinivasan et al. applied game theory and Nash equilibrium among the nodes to forward packets such that the total throughput (capacity) can achieve an optimal operating point subject to a common lifetime requirement on all nodes. However, the fairness issue in information collection was not considered. The most relevant work to ours is by Brown et al. [6], which has been discussed in detail in Section 4.1. 


\section{CONCLUSIONS}

In this paper, we investigated the important problem of rate allocation for wireless sensor networks under a given network lifetime requirement. Since the objective of maximizing the sum of rates of all nodes can lead to a severe bias in rate allocation among the nodes, we advocate the use of lexicographical max-min (LMM) rate allocation for all nodes in the network. To calculate the LMMoptimal rate vector, we developed a polynomial-time algorithm by exploiting the parametric analysis (PA) technique from linear programming (LP), which we called serial LP with Parametric Analysis (SLP-PA). Furthermore, we showed that the SLP-PA algorithm can also be employed to address the maximum node lifetime curve problem and that the SLP-PA algorithm is much more efficient than existing techniques. More importantly, we discovered a simply and elegant duality relationship between the LMM rate allocation problem and LMM node lifetime problem, which enable us to develop solutions and insights on both problems by solving any one of the two problems. Our results in this paper offer some important understanding on network capacity and network lifetime problems for energy-constrained wireless sensor networks.

Our efforts in this paper have been centered on developing centralized theory on the LMM rate allocation problem and its relationship to the LMM node lifetime problem. This understanding is essential to further research on distributed implementations, which are currently underway and the results of which will be reported in a separate paper.

\section{ACKNOWLEDGEMENT}

Y.T. Hou wishes to thank Zixiang Xiong of Texas A\&M Univ. for discussions on distributed source coding (DSC) paradigm for information processing in sensor networks.

\section{REFERENCES}

[1] I.F. Akyildiz, W. Su, Y. Sankarasubramaniam, and E. Cayirci, "Wireless sensor networks: A survey," Computer Networks (Elsevier), vol. 38, no. 4, pp. 393-422, 2002.

[2] M.S. Bazaraa, J.J. Jarvis, and H.D. Sherali, Linear Programming and Network Flows, second edition, Chapters 4, 6, and 8, John Wiley \& Sons, 1990.

[3] D. Bertsekas and R. Gallager, Data Networks, Chapter 6, Prentice Hall, 1992.

[4] M. Bhardwaj and A.P. Chandrakasan, "Bounding the lifetime of sensor networks via optimal role assignments," in Proc. IEEE Infocom, pp. 1587-1596, June 23-27, 2002, New York, NY.

[5] D. Blough and S. Paolo, "Investigating upper bounds on network lifetime extension for cell-based energy conservation techniques in stationary ad hoc networks," in Proc. ACM MobiCom, pp. 183-192, Sept. 23-28, 2002, Atlanta, GA.

[6] T.X. Brown, H.N. Gabow, and Q. Zhang, "Maximum flow-life curve for a wireless ad hoc network," in Proc. ACM MobiHoc, pp. 128-136, Oct. 4-5, 2001, Long Beach, CA.

[7] J.-H. Chang and L. Tassiulas, "Routing for maximum system lifetime in wireless ad-hoc networks," in Proc. 37th Annual Allerton Conference on Communications, Control, and Computing, Sept. 1999, Monticello, IL.
[8] J.-H. Chang and L. Tassiulas, "Energy conserving routing in wireless ad-hoc networks," in Proc. IEEE Infocom, pp. 22-31, March 26-30, 2000, Tel Aviv, Israel.

[9] J. Chou, D. Petrovis, and K. Ramchandran, "A distributed and adaptive signal processing approach to reducing energy consumption in sensor networks," in Proc. IEEE Infocom, pp. 1054-1062, April 1-3, 2003, San Francisco, CA.

[10] W. Heinzelman, Application-specific Protocol Architectures for Wireless Networks, Ph.D. thesis, MIT, 2000.

[11] Y.T. Hou, Y. Shi, and H.D. Sherali, "On rate allocation problem for wireless sensor networks," Technical Report, The Bradley Department of Electrical and Computer Engineering, Virginia Tech, July 2003. Available at http: //www. ece.vt. edu/ thou/Research.

[12] K. Kar, M. Kodialam, T.V. Lakshman, and L. Tassiulas, "Routing for network capacity maximization in energy-constrained ad-hoc networks," in Proc. IEEE Infocom, pp. 673-681, April 1-3, 2003, San Francisco, CA.

[13] K. Kalpakis, K. Dasgupta, and P. Namjoshi, "Maximum lifetime data gathering and aggregation in wireless sensor networks," in Proc. IEEE International Conference on Networking (ICN'02), pp. 685-696, Aug. 26-29, 2002, Atlanta, GA.

[14] H. Luss and D.R. Smith, "Resource allocation among competing activities: a lexicographic minimax approach," Operations Research Letters, vol. 5, no. 5, pp. 227-231, Nov. 1986.

[15] S.S. Pradhan, J. Kusuma, and K. Ramchandran, "Distributed compression in a dense sensor network," IEEE Signal Processing Magazine, vol. 19, no. 2, pp. 51-60, March 2002.

[16] R. Ramanathan and R. Rosales-Hain, "Topology control of multihop wireless networks using transmit power adjustment," in Proc. IEEE Infocom, pp. 404-413, March 26-30, 2000, Tel Aviv, Israel.

[17] K. Ramchandran, "Distributed sensor networks: opportunities and challenges in signal processing and communications," presentation at NSF Workshop on Distributed Communications and Signal Processing for Sensor Networks, Dec. 2002, Evanston, IL. Available at http: //www.ece.northwestern.edu/ pappas /nsf_workshop/presentations/ramchandran _workshop_DDSP.ppt.

[18] T.S. Rappaport, Wireless Communications: Principles and Practice, Prentice Hall, New Jersey, 1996.

[19] V. Rodoplu and T.H. Meng, "Minimum energy mobile wireless networks," IEEE Journal on Selected Areas in Communications, vol. 17, no. 8, pp. 1333-1344, Aug. 1999.

[20] V. Srinivasan, P. Nuggehalli, C.F. Chiasserini, and R. Rao, "Cooperation in wireless ad hoc networks," in Proc. IEEE Infocom, pp. 808-817, April 1-3, 2003, San Francisco, CA.

[21] R. Wattenhofer, L. Li, P. Bahl, and Y.-M. Wang, "Distributed topology control for power efficient operation in multihop wireless ad hoc networks," in Proc. IEEE Infocom, pp. 1388-1397, April 22-26, 2001, Anchorage, AK.

[22] G. Zussman and A. Segall, "Energy efficient routing in ad hoc disaster recovery networks," in Proc. IEEE Infocom, pp. 405-421, April 1-3, 2003, San Francisco, CA. 